\title{
An Index for T-wave Pointwise Amplitude Variability Quantification
}

\author{
Julia Ramírez ${ }^{1,2}$, Michele Orini ${ }^{3}$, J. Derek Tucker ${ }^{4}$, Esther Pueyo $^{2,1}$ and Pablo Laguna ${ }^{2,1}$ \\ ${ }^{1}$ Biomedical Research Networking Center in Bioengineering, Biomaterials and Nanomedicine \\ (CIBER-BBN), Zaragoza, Spain. \\ ${ }^{2}$ Biomedical Signal Interpretation and Computational Simulation (BSICoS) group, Aragón Institute \\ of Engineering Research (I3A), IIS Aragón, University of Zaragoza, Zaragoza, Spain. \\ ${ }^{3}$ Institute of Cardiovascular Science, University College London, London, UK. \\ ${ }^{4}$ Sandia National Laboratories, Albuquerque, NM, USA.
}

\begin{abstract}
The comparison between the pointwise amplitude of different $T$-waves provides insight into ventricular repolarization liability. However, T-wave pointwise amplitude variability can be confounded by time-domain variability. We, first, compared two algorithms for removing (warping) time-domain variability, one using the original and another one using a transformed T-wave (SRSF). We, next, compared the robustness against noise of two markers, $d_{y}$ and $d_{a}$, of pointwise amplitude variability, after warping the underlying temporal variability with the preferred warping algorithm. $d_{y}$ was obtained from the transformed $T$-waves while $d_{a}$ was proposed in this work and was derived from the original $T$-waves. We, finally, used the most robust marker to measure the T-wave pointwise amplitude variability between every $T$-wave recorded during a Tilt test and their mean T-wave. Results showed that the preferred warping algorithm was the SRSF because it is not affected by differences between the amplitudes of the original T-waves. In addition, the marker $d_{a}$ presented lower relative error values than $d_{y}$ for every level of noise. The analysis of electrocardiogram records showed that $d_{a}$ was significantly lower during the tilt than in supine position ($5.5 \%$ vs $6.5 \%, p<0.01)$. In conclusion, $d_{a}$ robustly quantifies physiological variabilities of the T-wave amplitude, showing its potential to be used as an arrhythmic risk predictor in future clinical situations.
\end{abstract}

\section{Introduction}

The T-wave on the electrocardiogram (ECG) reflects the spatio-temporal repolarization heterogeneity of the ventricular myocardium and its duration and amplitude are commonly used to diagnose pathologies and assess risk of malignant arrhythmias [1]. Variabilities in such spatiotemporal repolarization heterogeneities are associated with increased arrhythmic risk [2], and this motivates the quantification of the variations of the amplitude of the T- wave. However, those repolarization heterogeneities, or other physiological situations like changes in the heart rate, might induce variabilities in the temporal domain (such as stretches and translations of the T-wave) that need to be separated (warped) from the variability along the amplitude domain. This motivates the seek for a robust marker of T-wave pointwise amplitude variability, independent from the underlying time-domain variability. The most traditional algorithm for time-domain warping is the dynamic time warping (DTW) [3], which performs a sampleto-sample projection of two T-waves that aims at minimizing the Euclidean distance between them. DTW leads to a warping function that can be used to remove the time domain variability present in the original T-waves. Recently, a variation of DTW based on a mathematical representation of the signals, called the "square-root slope function" (SRSF), was proposed $[4,5]$. The SRSF transformation improves alignment and provides fundamental mathematical equalities that lead to a formal development of the warping problem. In this framework, a marker of amplitude variability, $d_{y}$, is defined as the Euclidean distance of the difference between the SRSFs of the reference and the warped $\mathrm{T}$-waves.

The first objective of this study is to compare the performances of the DTW and SRSF warping algorithms in removing time-domain variability. Next, we propose a biomarker of T-wave pointwise amplitude variability, $d_{a}$, and compare its robustness against additive Laplacian noise with that from $d_{y}$, after warping the underlying temporal variability with the preferred warping algorithm. Finally, we use the marker with higher robustness to measure the pointwise amplitude variability induced by a Tilt test.

\section{Methods}

\subsection{Amplitude Variability Quantification}

Consider $\boldsymbol{f}^{r}\left(\boldsymbol{t}^{r}\right)=\left[f^{r}\left(t^{r}(1)\right), \ldots, f^{r}\left(t^{r}\left(N_{r}\right)\right)\right]^{T}$ and $\boldsymbol{f}^{s}\left(\boldsymbol{t}^{s}\right)=\left[f^{s}\left(t^{s}(1)\right), \ldots, f^{s}\left(t^{s}\left(N_{s}\right)\right)\right]^{T}$, as two T- 
waves, where $\boldsymbol{t}^{r}=\left[t^{r}(1), \ldots, t^{r}\left(N_{r}\right)\right]^{T}$ and $\boldsymbol{t}^{s}=$ $\left[t^{s}(1), \ldots, t^{s}\left(N_{s}\right)\right]^{T}$, and $N_{r}$ and $N_{s}$ are the total duration of $\boldsymbol{t}^{r}$ and $\boldsymbol{t}^{s}$, respectively. We take $\boldsymbol{f}^{r}\left(\boldsymbol{t}^{r}\right)$ as the reference T-wave and $\boldsymbol{f}^{s}\left(\boldsymbol{t}^{s}\right)$ as the T-wave to be compared with respect to $\boldsymbol{f}^{r}\left(\boldsymbol{t}^{r}\right)$.

Let $\gamma\left(\boldsymbol{t}^{r}\right)$ be the warping function that relates $\boldsymbol{t}^{r}$ and $\boldsymbol{t}^{s}$, such that the composition $\boldsymbol{f}^{s}\left(\gamma\left(\boldsymbol{t}^{r}\right)\right)$ denotes the reparameterization or time domain warping of $\boldsymbol{f}^{s}\left(\boldsymbol{t}^{s}\right)$ using $\gamma\left(\boldsymbol{t}^{r}\right)$, i.e. $\boldsymbol{f}^{s}\left(\gamma\left(\boldsymbol{t}^{r}\right)\right)$ represents the amplitude values of $\boldsymbol{f}^{s}\left(\boldsymbol{t}^{s}\right)$ if its temporal vector was $\boldsymbol{t}^{r}$. Then, the DTW algorithm finds the optimal warping function, $\gamma_{\mathrm{w}}^{*}\left(\boldsymbol{t}^{r}\right)$, as:

$$
\boldsymbol{\gamma}_{\mathrm{w}}^{*}\left(\boldsymbol{t}^{r}\right)=\underset{\gamma\left(\boldsymbol{t}^{r}\right)}{\arg \min }\left(\left\|\boldsymbol{f}^{r}\left(\boldsymbol{t}^{r}\right)-\boldsymbol{f}^{s}\left(\boldsymbol{\gamma}\left(\boldsymbol{t}^{r}\right)\right)\right\|\right) .
$$

The SRSF of a T-wave $\boldsymbol{f}(\boldsymbol{t})$ is defined in the following form $[4,5]$ :

$$
\boldsymbol{q}_{f}(\boldsymbol{t})=\operatorname{sign}(\dot{\boldsymbol{f}}(\boldsymbol{t})) \cdot \sqrt{|\dot{\boldsymbol{f}}(\boldsymbol{t})|}
$$

If we warp a $\boldsymbol{f}(\boldsymbol{t})$ by $\gamma(\boldsymbol{t})$, the SRSF of $\boldsymbol{f}(\gamma(\boldsymbol{t}))$ is given by: $\boldsymbol{q}_{f}(\gamma(\boldsymbol{t})) \cdot \sqrt{\dot{\gamma}(\boldsymbol{t})}$. Now, the optimal warping function was proposed in $[4,5]$ as the function that minimizes the Euclidean distance of the difference between the SRSF of the original signals, obtaining a transformed warping function, denoted as $\boldsymbol{\gamma}_{\mathrm{TW}}^{*}\left(\boldsymbol{t}^{r}\right)$ :

$\boldsymbol{\gamma}_{\mathrm{TW}}^{*}\left(\boldsymbol{t}^{r}\right)=\underset{\boldsymbol{\gamma}\left(\boldsymbol{t}^{r}\right)}{\arg \min }\left(\left\|\boldsymbol{q}_{f^{r}}\left(\boldsymbol{t}^{r}\right)-\boldsymbol{q}_{f^{s}}\left(\boldsymbol{\gamma}\left(\boldsymbol{t}^{r}\right)\right) \cdot \sqrt{\dot{\gamma}\left(\boldsymbol{t}^{r}\right)}\right\|\right)$.

Using the optimal warping function we can define two markers of pointwise amplitude variability, that are independent from the underlying time-domain variability:

$$
\begin{aligned}
& d_{y}=\operatorname{sign}\left(e_{y}\right) \cdot \frac{\overbrace{\boldsymbol{q}_{f^{r}}\left(\boldsymbol{t}^{r}\right)-\boldsymbol{q}_{\boldsymbol{f}^{s}}\left(\boldsymbol{\gamma}_{\mathrm{TW}}^{*}\left(\boldsymbol{t}^{r}\right)\right) \cdot \sqrt{\dot{\boldsymbol{\gamma}}_{\mathrm{TW}}^{*}\left(\boldsymbol{t}^{r}\right)}}^{\left\|\boldsymbol{q}_{f^{r}}\left(\boldsymbol{t}^{r}\right)\right\|}}{\boldsymbol{v}_{y}} \times 100, \\
& e_{y}=\sum_{n=1}^{N_{r}} v_{y}(n) . \\
& d_{a}=\operatorname{sign}\left(e_{a}\right) \cdot \frac{\|\overbrace{\boldsymbol{f}^{r}\left(\boldsymbol{t}^{r}\right)-\boldsymbol{f}^{s}\left(\boldsymbol{\gamma}_{\mathrm{TW}}^{*}\left(\boldsymbol{t}^{r}\right)\right)}^{\boldsymbol{v}_{a}}\|}{\left\|\boldsymbol{f}^{r}\left(\boldsymbol{t}^{r}\right)\right\|} \times 100, \\
& e_{a}=\sum_{n=1}^{N_{r}} v_{a}(n) .
\end{aligned}
$$

\subsection{Signal Preprocessing}

Preprocessing of the ECG signals included low-pass filtering at $40 \mathrm{~Hz}$ to remove electric and muscle noise but to allow QRS detection, cubic splines interpolation for baseline wander removal and ectopic beats detection. Principal Component Analysis was calculated lead-wise over the Twaves from the available leads to emphasize the T-wave energy, improve its delineation and enhance morphological differences [6].
Each T-wave from the first principal component was selected using the T-wave onset and T-wave offset delineation marks [7]. Then, each T-wave was further low-pass filtered at $20 \mathrm{~Hz}$ to remove the components that could potentially corrupt the T-wave shape.

\subsection{Simulation Study}

The accuracy of the different markers of T-wave amplitude variability, $d_{y}$ and $d_{a}$, in detecting T-wave amplitude variations was assessed by simulating, under the presence of different levels of additive noise, controlled variations in the T-wave duration and amplitude.

Let the T-wave from a reference noise-free cardiac beat, sampled at $1 \mathrm{kHz}$, be the reference T-wave, $\boldsymbol{f}^{r}\left(\boldsymbol{t}^{r}\right)$.

T-wave amplitude variability was modelled by multiplying the deviations from the iso-electric line of each $i$-th $\mathrm{T}$-wave by a sinusoidally evolving factor in the following way:

$$
\begin{aligned}
\boldsymbol{f}_{i}^{s}\left(\boldsymbol{t}^{r}\right) & =\boldsymbol{f}^{r}\left(\boldsymbol{t}^{r}\right) \cdot\left(1+0.25 \cdot \sin \left(\frac{\pi(i-1)}{I-1}\right)\right), \\
i & =1, \ldots, I,
\end{aligned}
$$

T-wave time domain modulation was introduced by modifying the temporal domain of $\boldsymbol{f}^{r}\left(\boldsymbol{t}^{r}\right)$ according to the following equation:

$$
\begin{aligned}
\boldsymbol{t}_{i}^{s} & =t^{r}(1)+\left(t^{r}\left(N_{r}\right)-t^{r}(1)\right) \cdot\left(\frac{\boldsymbol{t}^{r}-t^{r}(1)}{t^{r}\left(N_{r}\right)-t^{r}(1)}\right)^{\alpha(i)} \\
\alpha(i) & =\left(\frac{0.45(i-1)}{I-1}+0.8\right), \quad i=1, \ldots, I
\end{aligned}
$$

where $i$ indexes each heart beat, and $I$ is the total number of modulated beats. The $i$-th modulated cardiac beat was obtained by transforming $\boldsymbol{f}^{r}\left(\boldsymbol{t}^{r}\right)$ to $\boldsymbol{f}_{i}^{s}\left(\boldsymbol{t}_{i}^{s}\right)$. Then, a simulated ECG signal was obtained by concatenating the $I=300$ modulated cardiac beats following the reference cardiac beat. This led to a 301-beat ECG signal. This simulated ECG signal was preprocessed, QRS detected and delineated, and the T-waves were delimited as explained in section 2.2. The reference $\boldsymbol{d}_{a}^{r}=\left[d_{a}^{r}(1), \ldots, d_{a}^{r}(I)\right]$ and $\boldsymbol{d}_{y}^{r}=\left[d_{y}^{r}(1), \ldots, d_{y}^{r}(I)\right]$ series were obtained by warping each $\boldsymbol{f}_{i}^{s}\left(\boldsymbol{t}_{i}^{s}\right)$ with respect to $\boldsymbol{f}^{r}\left(\boldsymbol{t}^{r}\right)$, using (3) and applying eqs. (4) and (5).

Then, zero mean Laplacian noise was iteratively added to the simulated ECG signal, such that the signal-to-noise ratio (SNR) was, in decibels $(\mathrm{dB}): \mathrm{SNR}=\{5,10, \ldots, 35\}$. The estimated $\boldsymbol{d}_{a}^{\mathrm{SRR}}=\left[d_{a}^{\mathrm{SNR}}[1], \ldots, d_{a}^{\mathrm{SNR}}[I]\right]$ and $\boldsymbol{d}_{y}^{\mathrm{SNR}}=$ $\left[d_{y}^{\mathrm{SNR}}[1], \ldots, d_{y}^{\mathrm{SNR}}[I]\right]$ series were obtained by comparing the T-waves from the noisy modulated ECG signal with the noisy reference T-wave, located in the first beat. The estimation errors were, then, calculated as:

$$
e_{d}(\mathrm{SNR})=\sqrt{\frac{\sum_{i=1}^{I}\left(d^{\mathrm{SNR}}(i)-d^{r}(i)\right)^{2}}{\sum_{i=1}^{I}\left(d^{r}(i)\right)^{2}}} \times 100,
$$



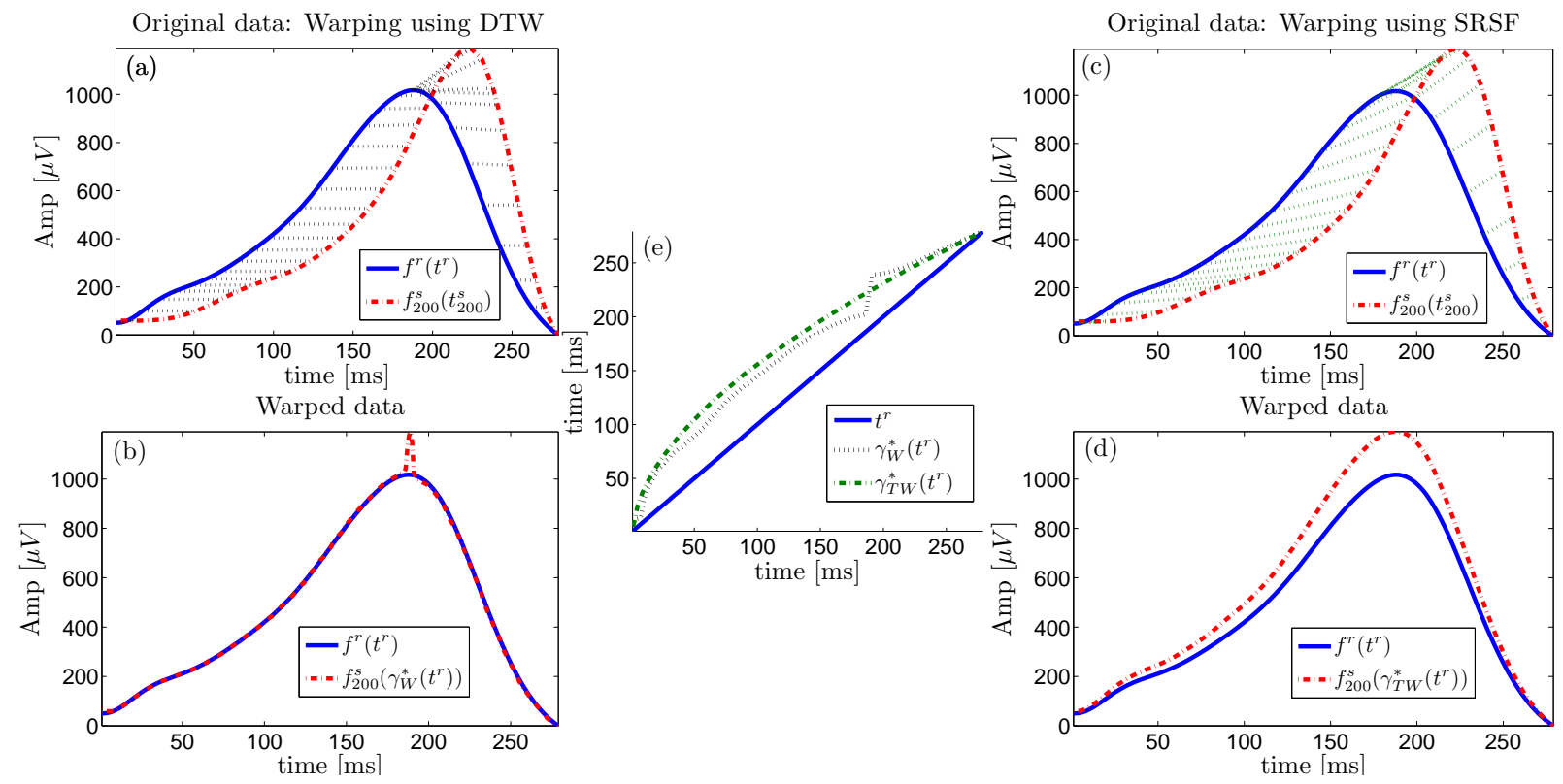

Figure 1. Comparison of DTW and SRSF warping algorithms. Panels (a) and (c) show $\boldsymbol{f}^{r}\left(\boldsymbol{t}^{r}\right)$ (solid blue) and $\boldsymbol{f}_{200}^{s}\left(\boldsymbol{t}_{200}^{s}\right)$ (dashed red). The dotted lines illustrate the relation between $\boldsymbol{t}^{r}$ and $\boldsymbol{t}^{s}$ using $\boldsymbol{\gamma}_{\mathrm{W}}^{*}\left(\boldsymbol{t}^{r}\right)$ and $\boldsymbol{\gamma}_{\mathrm{TW}}^{*}\left(\boldsymbol{t}^{r}\right)$, respectively (plotted in panel (e)). Panels (b) and (d) show $\boldsymbol{f}_{200}^{s}\left(\boldsymbol{\gamma}_{\mathrm{W}}^{*}\left(\boldsymbol{t}^{r}\right)\right)$ and $\boldsymbol{f}_{200}^{s}\left(\boldsymbol{\gamma}_{\mathrm{TW}}^{*}\left(\boldsymbol{t}^{r}\right)\right)$, respectively.

where $d=\left\{d_{y}, d_{a}\right\}$. The noise generation and relative error measurement steps were repeated 50 times in order to have robust relative error values.

\subsection{Real ECG}

ECG recordings from a database acquired at the University of Zaragoza for the study of the autonomic nervous system (ANS-UZ) were analyzed [8]. Recordings were obtained from 17 healthy subjects (age 28.5 \pm 2.8 years, 11 males) with no previous medical history related to cardiovascular diseases. Each recording consisted of 8 ECG leads, sampled at $1 \mathrm{KHz}$, acquired during a 13-min headup tilt test (4-min supine, 5-min at 70 ${ }^{\circ}$, 4-min supine).

The ECG recordings were preprocessed and delineated and $d_{a}$ series were obtained for each subject by comparing each T-wave with the mean of the T-waves in each ECG recording.

We assumed stationariness in three intervals, early supine, Tilt, and late supine, as done in [9]. These windows had a length of 20 beats and finished $30 \mathrm{~s}$ before any transition during the tilt test. The statistical significance between the median $R R$ values and the median of $d_{a}$, calculated within each interval, was evaluated using the Wilcoxon signedrank test.

\section{Results and discussion}

\subsection{Simulation Study}

Figure 1 shows an example of DTW (eq. (1)) and SRSF (eq. (3)) warping algorithms for $i=200$ in the simulation study. Panel (a) shows $\boldsymbol{f}^{r}\left(\boldsymbol{t}^{r}\right)$ (solid blue) and $\boldsymbol{f}_{200}^{s}\left(\boldsymbol{t}_{200}^{s}\right)$ (dashed red), where the simulated amplitude scaling (by a factor of 1.2) and temporal asymmetry $(\alpha(200)=1.1)$ can be appreciated. The warping algorithm is expected to selectively compensate for the time-domain variability, so the resulting warped T-wave should be a scaled version of $\boldsymbol{f}^{r}\left(\boldsymbol{t}^{r}\right)$. However, this is obtained only using SRSF (right panels), because DTW (left panels) jointly compensates for time and amplitude, providing a warped T-wave different from the expected one. The black dotted lines illustrate the pointwise warping reflected by $\gamma_{\mathrm{w}}^{*}\left(\boldsymbol{t}^{r}\right)$ (depicted on panel (e)). The resulting warped $\boldsymbol{f}_{200}^{s}\left(\boldsymbol{\gamma}_{\mathrm{W}}^{*}\left(\boldsymbol{t}^{r}\right)\right)$ is shown in panel (b). It can be observed how DTW produces a singularity, or unintuitive warping, leading to a degenerate warped T-wave. This occurs because DTW compares amplitude values, rather than match the features of both T-waves (up-/downslopes, peaks, etc). For instance, in panel (a), $\gamma_{\mathrm{w}}^{*}\left(\boldsymbol{t}^{r}\right)$ has matched the peak of $\boldsymbol{f}^{r}\left(\boldsymbol{t}^{r}\right)$ to the first point found in $\boldsymbol{f}_{200}^{s}\left(\boldsymbol{t}_{200}^{s}\right)$ with the same amplitude value, without considering if this point is also the peak. Panels (c) and (d) show the same process, but using the SRSF warping algorithm. SRSF is based on the comparison of two transformed functions that are proportional to the derivative of the original signals so that, together with its built-in regularization term, $\sqrt{\dot{\gamma}_{\text {TW }}^{*}\left(\boldsymbol{t}^{r}\right)}$, SRSF achieves a feature-to-feature warping (green dotted lines, panels (c) and (e)), leading to a warped $\boldsymbol{f}_{200}^{s}\left(\boldsymbol{\gamma}_{\mathrm{TW}}^{*}\left(\boldsymbol{t}^{r}\right)\right)$ that is just a scaled version of $\boldsymbol{f}^{r}\left(\boldsymbol{t}^{r}\right)$, as expected. Therefore, we will use $\gamma_{\mathrm{TW}}^{*}\left(\boldsymbol{t}^{r}\right)$ as the optimal warping function in the rest of this document.

Figure 2 shows the mean \pm standard deviation of the relative error between $d_{a}^{\mathrm{SNR}}$ and $d_{a}^{r}$ (blue), and $d_{y}^{\mathrm{SNR}}$ and $d_{y}^{r}$ (red), for different values of SNR. The relative error val- 


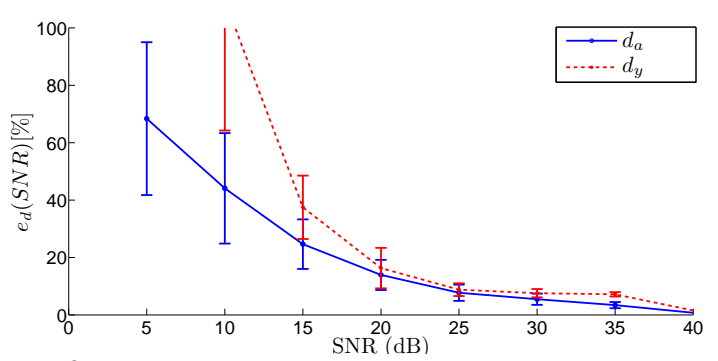

Figure 2. Relative error between reference and estimated $d_{a}$ (solid blue) and $d_{y}$ (dashed red) under the presence of additive Laplacian noise.

ues of $d_{y}$ are higher than those from $d_{a}$ for every value of $S N R$ (Figure 2). This is because the SRSF transformation used for the calculation of $d_{y}$ (eq. 4) highlights the high-frequency components of the signal, resulting in less robust estimates against additive broadband noise.

\subsection{Real ECG}

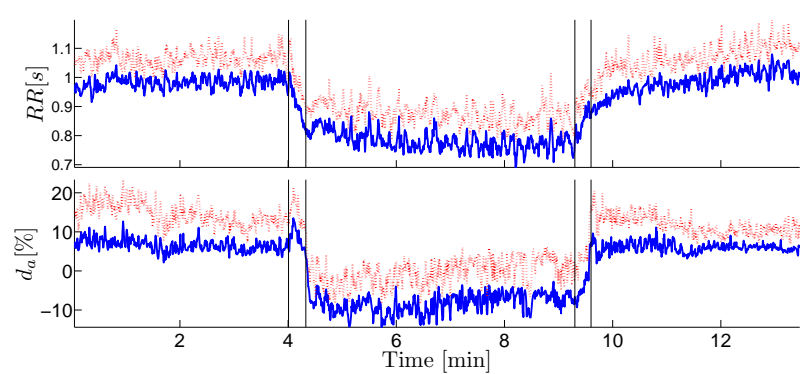

Figure 3. Median (blue) and median + median absolute deviation (red) of the $R R$ (top panel) and $d_{a}$ (bottom panel) series during a Tilt test, calculated across subjects. Vertical lines indicate the start and end of the change in the table tilt.

We used $d_{a}$ to measure the T-wave amplitude variability produced by a Tilt test, after warping its time-domain variability with $\boldsymbol{\gamma}_{\mathrm{TW}}^{*}\left(\boldsymbol{t}^{r}\right)$, since, as previously shown, $\boldsymbol{\gamma}_{\mathrm{TW}}^{*}\left(\boldsymbol{t}^{r}\right)$ efficiently removes the underlying time-domain variability, and $d_{a}$ is more robust against noise than $d_{y}$.

The median + median absolute deviation of the resulting $R R$ and $d_{a}$ series, calculated across subjects, are reported in Figure 3. It can be observed how the $d_{a}$ series follows the same dynamic as the $R R$ series, with negative values during the Tilt, showing that an orthostatic challenge provokes a reduction in the amplitude of the T-waves. Table 1 shows that the median values of $R R$ and $d_{a}$ were significantly smaller during the Tilt than during supine position.

\begin{tabular}{cccc}
\hline \hline & Early Supine & Tilt & Late Supine \\
\hline$R R[\mathrm{~s}]$ & $1.01(0.1)$ & $0.77(0.2)^{*}$ & $1.06(0.2)^{\dagger}$ \\
$d_{a}[\%]$ & $6.54(14.5)$ & $-5.54(22.6)^{*}$ & $6.56(6.9)^{\dagger}$ \\
\hline
\end{tabular}

* Indicates significantly different $(\mathrm{p}<0.01)$ with respect to Early Supine.

$\dagger$ Indicates significantly different $(\mathrm{p}<0.01)$ with respect to Tilt.

Table 1. Temporal evolution of the median (interquartile range), calculated across subjects, of $R R$ and $d_{a}$ during a Tilt test.

\section{Conclusions}

The present paper proposes a novel bio-marker for Twave pointwise amplitude variability quantification, $d_{a}$, independent from the underlying time domain variability. The index, $d_{a}$, was proven to be robust against noise and showed that significant changes occur in the pointwise $\mathrm{T}$ wave amplitude during head-up tilt.

The predictive value of this new marker of T-wave variability will be assessed in future studies to determine whether it may improve or complement existing markers $[2,10]$.

\section{Acknowledgements}

This work was supported in part by project TIN2013-41998-R from Spanish Ministry of Economy and Competitiveness (MINECO), Spain, and by Aragón Government, Spain and from European Social Fund (EU) through BSICoS group. The computation was performed at the High Performance computing platform of the NANBIOSIS ICTS, CIBER-BBN and I3A, Zaragoza, Spain. J. Ramírez acknowledges the contribution of D. Hernando in reviewing this document.

\section{References}

[1] M. J. Burgess. "Relation of ventricular repolarization to electrocardiographic $\mathrm{T}$ wave-form and arrhythmia vulnerability." Am J Physiol: Heart Circ Physiol vol. 5, pp.H391-H402, 1979.

[2] M. Baumert, et al. "QT interval variability in body surface ECG: measurement, physiological basis, and clinical value: position statement and consensus guidance endorsed by the European Heart Rhythm Association jointly with the ESC Working Group on Cardiac Cellular Electrophysiology." Europace 2016; doi:10.1093/europace/euv405.

[3] T. K. Vintsyuk. "Speech discrimination by dynamic programming." Cybernetics vol. 4, pp.52-57, 1968.

[4] A. Srivastava, et al, "Registration of Functional Data Using Fisher-Rao metric", arXiv:1103.3817v2[math.ST], 2011.

[5] J. D. Tucker, W. Wu and A. Srivastava. "Generative models for functional data using phase and amplitude separation." Computational Statistics and Data Analysis vol. 61, pp. 50-66, 2013.

[6] F. Castells, et al. "Principal component analysis in ECG signal processing." EURASIP Journal on Applied Signal Processing vol. 2007, pp.98-119, 2007.

[7] J. P. Martínez, et al. "A wavelet-based ECG delineator: evaluation on standard databases." IEEE Trans Biomed Eng vol. 51, pp.570-581, 2004.

[8] A. Mincholé, et al. "Quantification of restitution dispersion from the dynamic changes of the T-wave peak to end, measured at the surface ECG." IEEE Trans Biomed Eng vol. 58, no. 5, pp.1172-1182, May. 2011

[9] E. Gil, et al. "Photoplethysmography pulse rate variability as a surrogate measurement of heart rate variability during non-stationary conditions." Physiol Meas vol. 31, pp.1271-1290, 2010.

[10] J. Ramírez, et al. "Automatic SVM classification of sudden cardiac death and pump failure death from autonomic and repolarization ECG markers." J Electrocardiol vol. 48, pp.551-557, 2015.

Address for correspondence:

Julia Ramírez, Campus Río Ebro, I+D Building, D-5.01.1B, $\mathrm{c} \backslash$ Mariano Esquillor, s/n, 50018 Zaragoza (Spain),

Julia.Ramirez@unizar.es 